\title{
Perineuronal Sulfated Proteoglycans and Cell Surface Glycoproteins in Adult and Newborn Mouse Brains, with Special Reference to Their Postnatal Developments
}

\author{
Takuro Murakami ${ }^{1}$, Tetsuro Murakami ${ }^{2}$, Luo Jia Hong ${ }^{1}$, Wei Dong $\mathrm{SU}^{1}$, Da Xun Pia0 ${ }^{1}$, \\ Nazira MahmuT ${ }^{1,3}$ and Aiji OHTSUKA ${ }^{1}$ \\ Departments of Anatomy ${ }^{1}$, Neurology ${ }^{2}$ and Bacteriology ${ }^{3}$, Okayama University School of Medicine, Okayama, Japan \\ Received July 22, 1997
}

\begin{abstract}
Summary. Sections of the retrosplenial cortex from adult and newborn mouse brains were observed with a light microscope. The retrosplenial cortex of the adult animals contained many neurons (10\% of the total), including some dark neurons, with perineuronal sulfated proteoglycans detectable with cationic iron colloid and aldehyde fuchsin. The retrosplenial cortex of the adult animals also contained many neurons $(10 \%$ of the total) with cell surface glycoproteins reactive to lectin Vicia villosa, soybean or Wisteria floribunda agglutinin. Double staining showed that the majority (75\%) of the neurons labeled with lectins were stained with cationic iron colloid, and that some $(25 \%)$ of them were not stained with this colloid. Double staining also showed that some $(25 \%)$ of the neurons stained with cationic iron colloid were not labeled with lectins. These findings indicate that the perineuronal sulfated proteoglycans are, at least partly, independent from the cell surface glycoproteins. Observations of the sections from the newborn animals revealed that the perineuronal sulfated proteoglycans were produced by the associated satellite astrocytes $3-4$ weeks after birth, and that the cell surface glycoproteins were produced by the associated nerve cells at earlier stages, or 2-3 weeks after birth. Dark neurons began to appear 3-4 weeks after birth. These dark neurons or their Golgi complexes were also reactive to lectins, suggesting the production of cell surface glycoproteins.
\end{abstract}

Our light and electron microscopic studies of tissue sections stained with cationic iron colloid and aldehyde fuchsin have revealed the occurrence of many neurons with intensely negatively charged surface coats or perineuronal sulfated proteoglycans in the human brain (MURAKAMI et al., 1993b, 1994b, 1995a, 1996b, 1997a) as well as in the brains of cows, cats, rats, mice and other animals, including some lower vertebrates such as frogs and fishes (MURAKAMI et al., 1993a, c, 1994a, 1995a, b, 1996a, b; TsubouchI, Y. et al., 1996). Similar neurons have also been reported by other authors who mainly studied rat and mouse brains with lectin labelings or immunohistochemical stainings (BRAUER et al., 1984; NAKAGAWA et al., 1986; HENDRY et al., 1988; KosAKA and WeIzMANn, 1989; Bertolotto et al., 1990; NAEgele and Katz, 1990; DRAKE et al., 1991; BIGNAMI et al., 1992; HÄRTIG et al., 1992, 1994; LÜTH et al., 1992; BRÜCKNER et al., 1993, 1996; Celio and BlÜMCKE, 1994; SEEGER et al., 1994; BlÜMCKE et al., 1995; DEROUICHE et al., 1996; RuosLahti, 1996).

Our light and electron microscopic studies of human, rat and mouse brains further showed that the perineuronal sulfated proteoglycans develop as the extracellular matrix after birth (MURAKAMI, 1994; TsubOUCHI, M. et al., 1996), and that they are fairly independent from the cell surface glycoproteins labeled with lectins (MURAKAMI et al., 1995a, b, 1996a; TsubOUCHI, Y. et al., 1996) and identical to the Golgi's reticular coating stained with silver nitrate (MURAKAMI et al., 1996a, b).

The present study reinvestigates the brains of adult and newborn mice (MURAKAMI, 1994), and identifies the cells producing the perineuronal sulfated proteoglycans and cell surface glycoproteins. It also confirms the appearance of dark neurons in the newborn animals. The retrosplenial cortex forms the material of this study, as this cortex contains many neurons with well developed perineuronal proteoglycans and cell surface glycoproteins (MURAKAMI et al., 1996) and is well reactive to the circadian rhythm (OHTSUKA and MURAKAMI, 1996). 


\section{MATERIALS AND METHODS}

Newborn (0-7 weeks after birth) and adult (16 weeks after birth) ICR mice were anesthetized with ethyl ether in the afternoon, when sleeping. They were then perfused through the ascending aorta with Ringer's solution and with a mixture of $4.0 \%$ paraformaldehyde and $2.5 \%$ glutaraldehyde in $0.1 \mathrm{M}$ cacodylate buffer ( $\mathrm{pH}$ 7.2). From these animals, 1-2 mm thick blocks frontally traversing the retrosplenial cortex were removed, and fixed in the paraformaldehyde and glutaraldehyde mixture for $6 \mathrm{~h}$ or longer. The fixed blocks were embedded in paraffin, and cut into $10-15 \mu \mathrm{m}$ thick sections. The sections were deparaffinized with xylene.

The deparaffinized sections were stained with cationic iron colloid at a $\mathrm{pH}$ value of 1.5 (MURAKAMI et al., 1986), aldehyde fuchsin (GOMORI, 1950), lectin Vicia villosa agglutinin (KosAKA and WEIZMANN, 1989), lectin soybean agglutinin (LÜTH et al., 1992) or lectin Wisteria floribunda agglutinin (Ḧ̈RTIG et al., 1992). Some sections were stained doubly with cationic iron colloid and aldehyde fuchsin or with lectin and cationic iron colloid (pH 1.5) (MURAKAMI et al., 1995).

The cationic iron colloid staining was demonstrated by Prussian blue reaction (MURAKAMI et al., 1986) or by treatment with protein silver and gold chloride (MURAKAMI et al., 1997b). In the latter demonstration, the cationic iron colloid-treated sections were successively immersed in a $1 \%$ aqueous solution of protein silver (Albumosesilber, Merck) for 2-3 h, distilled water for $3 \mathrm{~min}$, a $1 \%$ aqueous solution of gold chloride for 2-3 h, distilled water for $10 \mathrm{~min}$, and an aqueous mixture of $1 \%$ hydroquinone and $5 \%$ formalin for $10 \mathrm{~min}$.

In the aldehyde fuchsin staining, preliminary treatment with potassium permanganate was omitted. A highly concentrated solution containing $2-3 \%$ aldehyde fuchsin (FUJITA, 1959) was sometimes used.

In the lectin stainings, peroxidase activity was demonstrated with diaminobenzidine (NAKAGAWA et al., 1985; BRÜCKNER et al., 1996). Controls for lectinstained sections consisted of adjacent sections treated with phosphate buffer containing no agglutinin.

\section{RESULTS}

\section{Adult mice}

In the retrosplenial cortex of the sleeping animals, the neurons were mostly light, with a few dark ones.

Many neurons ( $10 \%$ of the total), including some dark neurons, possessed the perineuronal sulfated proteoglycans reactive to cationic iron colloid (Figs. 1,2) and aldehyde fuchsin (Fig. 3). The perineuronal sulfated proteoglycans could be doubly stained with cationic iron colloid and aldehyde fuchsin (Fig. 3 lower Inset). The proteoglycans covered the perikaryon as well as the main processes of the nerve cells (Figs. 1 Insets, 2, Inset). No intracellular structures of any neurons, including the neurons with proteoglycans, were reactive to cationic iron colloid and aldehyde fuchsin.

Satellite astrocytes were sometimes invested in the perineuronal sulfated proteoglycans of their associated neurons (Figs. 1 upper Inset, 2 Inset). However, no intracellular structures of these invested glial cells were reactive to cationic iron colloid or aldehyde fuchsin stainings.

Many neurons ( $10 \%$ of the total)-including some dark neurons -in the retrosplenial cortex possessed cell surface glycoproteins reactive to lectin Vicia villosa, soybean or Wisteria floribunda agglutinin. Double staining revealed that the majority (about $75 \%$ ) of the neurons labeled with these lectins were stained with cationic iron colloid, and that some (25\%) of them showed no reaction to cationic iron colloid (Fig. 4, Insets). The double staining also revealed that some $(25 \%)$ of the neurons stained with cationic iron colloid were not labeled with lectins (Fig. 4, Insets). No intracellular structures of any neurons were labeled with lectin Vicia villosa, soybean or Wisteria floribunda agglutinins (Fig. 4 Insets). In the control sections, no neurons, including their surface and intracellular structures, were labeled with these lectins.

\section{Newborn mice}

The animals were weaned 4 weeks after birth.

The dark neurons began to appear 3-4 weeks after birth (Fig. 5 upper-left Inset). No dark neuron was noted at the earlier stages, or 0-2 weeks after birth.

The perineuronal sulfated proteoglycans began to appear 3-4 weeks after birth (Figs. 5-7). At this stage, the proteoglycans were lightly reactive to cationic iron colloid (Figs. 5, 6) and to aldehyde fuchsin (Fig. 7). The proteoglycans rapidly thickened. Thus, at 5 weeks after birth, the proteoglycans were easily recognizable even at low magnification. From 0-2 weeks after birth, no neuron was reactive to cationic iron colloid or aldehyde fuchsin.

At 3-4 weeks after birth, small-sized satellite cells were markedly reactive to cationic iron colloid and aldehyde fuchsin (Figs. 5-7). In typical cases, the cytoplasm of these glial cells was intensely stained with cationic iron colloid and aldehyde fuchsin as if it 

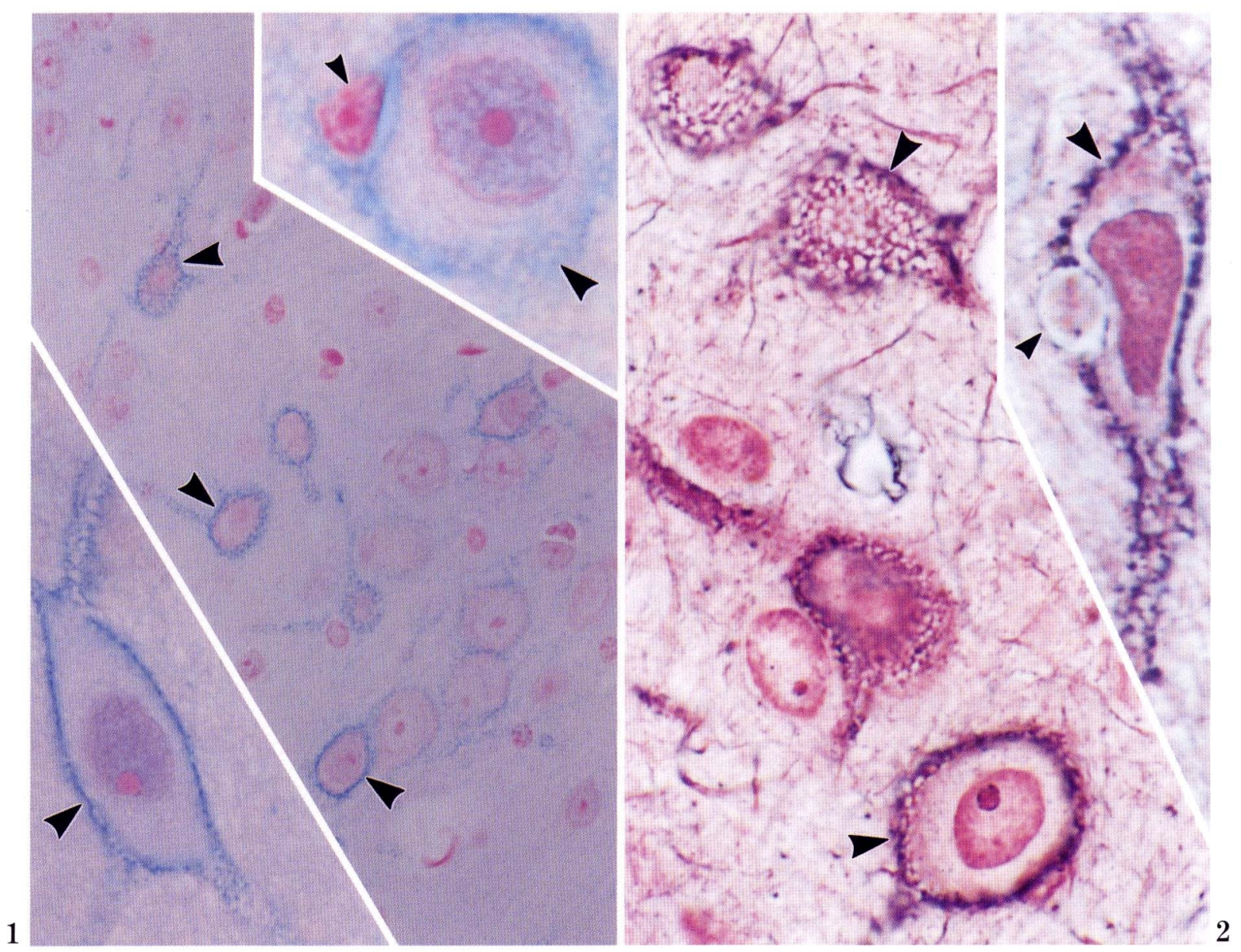

Fig. 1. Retrosplenial section from an adult mouse, stained with cationic iron colloid ( $\mathrm{pH}$ 1.5), treated for Prussian blue reaction and counter-stained with nuclear fast red. Many neurons are coated with perineuronal sulfated proteoglycans demonstrated by Prussian blue reaction (large arrowheads). Upper and lower Insets show closer views of the neurons with perineuronal sulfated proteoglycans (large arrowheads). The proteoglycans in the upper Inset invest an associated glial or satellite astrocyte (small arrowhead). $\times 500$, upper Inset: $\times 1,500$, lower Inset: $\times 1,100$

Fig. 2. Perineuronal sulfated proteoglycans, as stained with cationic iron colloid and demonstrated by treatment with protein silver and gold chloride (large arrowhead). Inset shows a neuron with perineuronal sulfated proteoglycans (large arrowhead) which invest an associated glial cell or satellite astrocyte (small arrowhead). $\times 1,100$, Inset: $\times 1,200$

was continuous with the sulfated proteoglycans covering the associated neuron (Fig. 6, Insets). This was most clearly noted in the specimens stained with aldehyde fuchsin, especially highly concentrated ones (Fig. 7, Insets). Such cytoplasmic staining of glial cells was not observed at 5 weeks or later stages after birth.

The cell surface glycoproteins detectable with lectin Vicia villosa, soybean or Wisteria floribunda agglutinin began to appear 2-3 weeks after birth (Fig. 8). The perinuclear areas or Golgi complexes of the neurons with these cell surface glycoproteins were always labeled with these lectins (Fig. 8 upper Inset). The Golgi complexes of the dark neurons with such cell surface glycoproteins were also always labeled with the lectins (Fig. 8 lower Inset). At the stage of $0-1$ week, no neuron was labeled with lectins. In the control sections, no neuron was reactive to diaminobenzidine.

The cell surface glycoproteins also developed rapidly. At 4 weeks after birth, the cell surfaces of many neurons were clearly and sharply labeled with lectin Vicia villosa, soybean and Wisteria floribunda agglutinins. At these stages, however, no intracellular or 

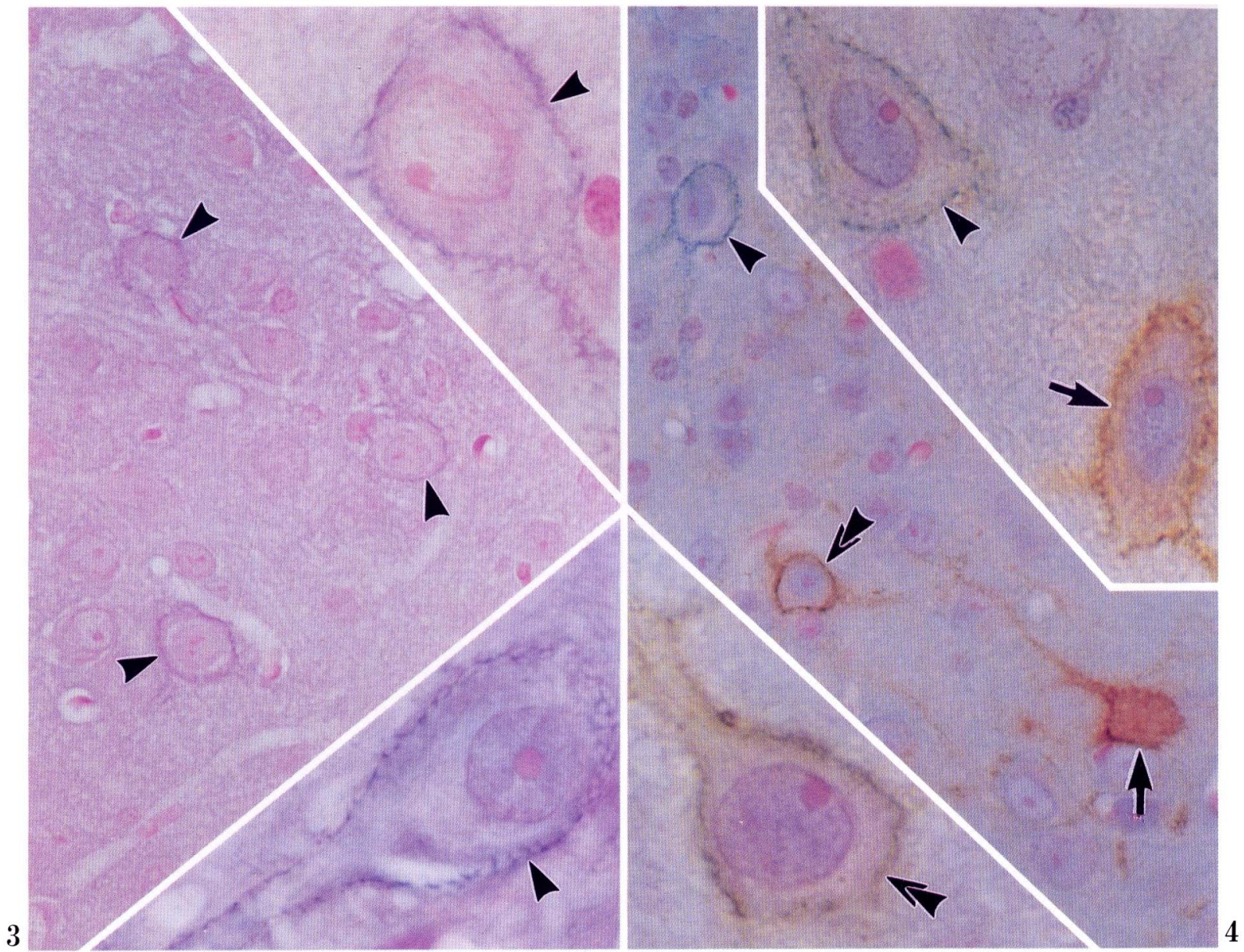

Fig. 3. Perineuronal sulfated proteoglycans, as stained with aldehyde fuchsin (large arrowheads). Upper Inset shows a closer view of a neuron with aldehyde fuchsin-stained perineuronal sulfated proteoglycans (large arrowhead). Lower Inset shows a closer view of the perineuronal proteoglycan-meshworks as doubly stained with cationic iron colloid (Prussian blue reaction) and aldehyde fuchsin (large arrowhead). $\times 600$, upper and lower Insets: $\times 1,300$

Fig. 4 and Insets. Retrosplenial sections doubly stained with cationic iron colloid (Prussian blue reaction) and lectin Vicia villosa agglutinin. Large arrowheads indicate the neurons solely reactive to cationic iron colloid. Large arrows indicate the neurons solely reactive to lectin. Double arrowheads indicate neurons doubly stained with cationic iron colloid and lectin. $\times 400$, upper Inset: $\times 1,100$, lower Inset: $\times 1,300$

perinuclear lectin labeling was noted in any cells, including the dark neurons.

It was also clearly confirmed in the doubly stained sections from the newborn animals 3-7 weeks after birth, that some of the lectin-labeled neurons were not stained with cationic iron colloid, and also that some of the neurons stained with cationic iron colloid were not labeled with lectins (data, not shown).

\section{DISCUSSION}

The present study, together with previous ones
(Murakami, 1994; Murakami et al., 1995b, 1996a), confirms that the mouse brain contains many neurons with intensely negatively charged surface coats or perineuronal sulfated proteoglycans which are stained with cationic iron colloid and aldehyde fuchsin. Our previous experiments with human, rat and mouse brains have shown that chondroitinase ABC/ heparitinase/keratanase digestions thoroughly erase the cationic iron colloid staining of the coats, though they never interfere with aldehyde fuchsin staining of the coats, indicating that cationic iron colloid and aldehyde fuchsin stain the sulfate groups and core proteins of proteoglycans, respectively (MURAKAMI, 


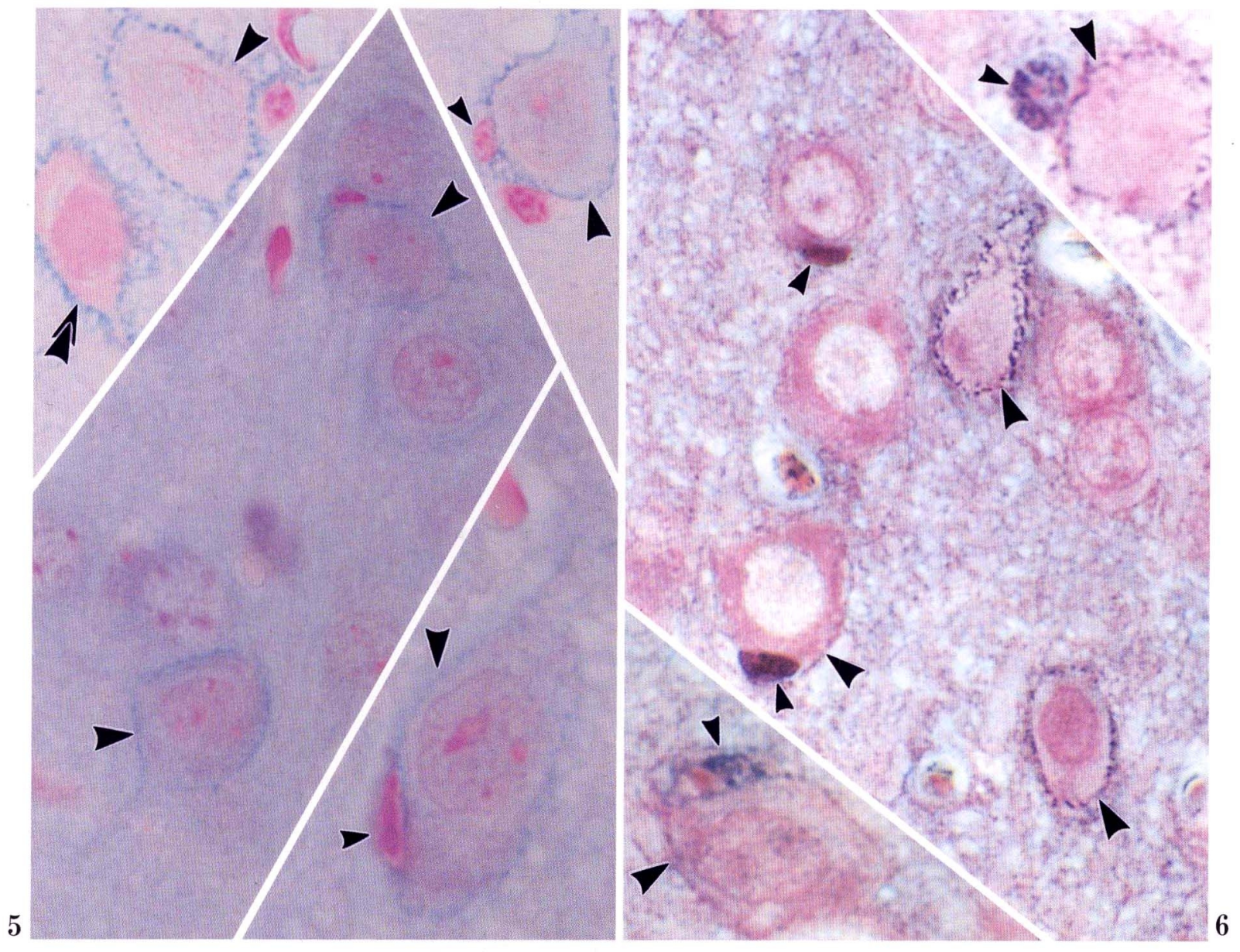

Fig. 5 and Insets. Retrosplenial sections from a newborn mouse 3 weeks after birth, stained with cationic iron colloid (Prussain blue reaction) and nuclear fast red. Some neurons are coated with perineuronal sulfated proteoglycans lightly stained with cationic iron colloid (large arrowheads). Upper-left Inset shows two neurons with perineuronal sulfated proteoglycans (arrowhead), one of which is darkened (double large arrowhead). Upper-right and lower Insets also show neurons with perineuronal sulfated proteoglycans (large arrowheads), which commonly invest or enclose associated satellite astrocytes (small arrowheads). $\times 1,000$, upper left and right Insets: $\times 1,100$, lower-right Inset: $\times 1,300$

Fig. 6 and Insets. Retrosplenial sections from another newborn mouse 3 weeks after birth, stained with cationic iron colloid and demonstrated by treatment with protein silver and gold chloride. Some satellite astrocytes are reactive to cationic iron colloid (small arrowheads) or show similar staining as the perineuronal sulfated proteoglycans (large arrowheads). Upper and lower Insets show neurons coated with developing perineuronal sulfated proteoglycans (large arrowheads). These proteoglycans are closely associated with the satellite astrocytes (small arrowheads). ×1,000, upper and lower Insets: $\times 1,200$

1994; Murakami et al., 1994a, b; Tsubouchi, Y., 1996).

The present double staining, in accordance with our previous results in human, rat and mouse brains (MURAKAMI et al., 1994b, 1995a, 1996a), clearly shows that some neurons labeled with lectin Vicia villosa, soybean or Wisteria floribunda agglutinin are not stained with cationic iron colloid, and also that some neurons stained with cationic iron colloid are not labeled with these lectins. Our previous experiments in the same milieus have shown that hyaluronidase digestion never interferes with the lectin labelings of the neurons though it thoroughly erases the cationic iron colloid and aldehyde fuchsin stainings of the neurons, indicating that the cell surface glycoproteins are independent from the perineuronal sulfated proteoglycans (MURAKAMI et al., 1995a, b, 1996a).

Our present and previous studies have thus added 


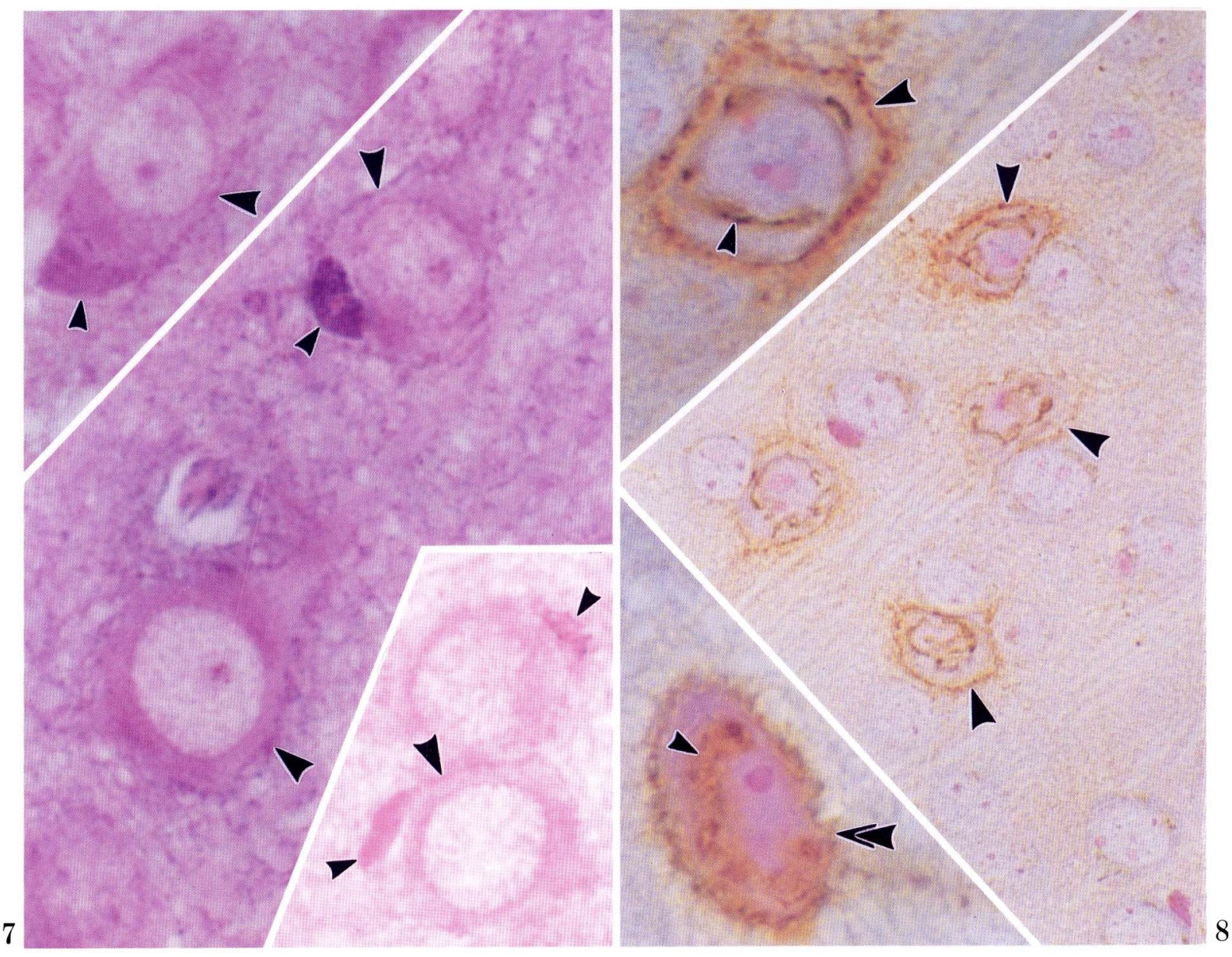

Fig. 7 and Insets. Retrosplenial sections from a newborn mouse 3 weeks after birth, stained with aldehyde fuchsin. Sections in the main figure and upper Inset were stained with concentrated aldehyde fuchsin. The cytoplasm of the satellite astrocytes are stained with aldehyde fuchsin (small arrowheads), together with the developing perineuronal sulfated proteoglycans (large arrowheads). $\times 1,300$, upper Inset: $\times 1,200$, lower Inset: $\times 1,300$

Fig. 8. Retrosplenial section from a newborn mouse 2 weeks after birth, labeled with lectin Vicia villosa agglutinin and counter-stained with nuclear fast red. The cell surface and Golgi complexes of some neurons are intensely labeled with this lectin (large arrowheads). Upper Inset shows a closer view of a light neuron labeled with lectin Vicia villosa agglutinin (large arrowhead). The Golgi complex is intensely labeled with lectin (small arrowhead), together with the cell surface (large arrowhead). Lower Inset shows a dark neuron (double large arrowhead) whose cell surface and Golgi complex (small arrowhead) are labeled with lectin Vicia villosa agglutinin. $\times 700$, Insets: $\times 1,400$

new insights to the results by LÜTH et al. (1992), BrüCKNER et al. (1993, 1996), CELIO and BLÜMCKE (1994), SEEGER et al. (1994) and other authors (DEROUI CHE et al., 1996) who have noted that neurons with cell surface glycoproteins are merely identical to neurons with perineuronal sulfated proteoglycans. Our previous observation of serial sections from the human brain has indicated that the neurons labeled with lectin Vicia villosa agglutinin are not always identical to the neurons labeled with lectin soybean agglutinin (MURAKAMI et al., 1995a). Such observations of lectin-labeled specimens (identifications of the lectin Wisteria floribunda agglutinin-labeled neurons with the lectin Vicia villosa or soybean agglutinin-labeled neurons) were omitted in the present study.

The present study, together with our previous ones in rat and mouse brains (MURAKAMI, 1994; TSUBOUCHI, M. et al., 1996), proves that the perineuronal sulfated proteoglycans and cell surface glycoproteins 
develop 2-4 weeks after birth. These suggest that the brain morphologically matures after birth or at the weaning period.

It is noteworthy that in newborn animals, the cytoplasm of some satellite astrocytes shows intense reactions to cationic iron colloid and aldehyde fuchsin which preferentially stain the perineuronal sulfated proteoglycans. This finding suggests that these glial cells produce the perineuronal sulfated proteoglycans. Decreased reactions of the glial cells to cationic iron colloid and aldehyde fuchsin after weaning may suggest that the proteoglycans are only poorly produced in these cells in adult animals. Our findings, thus, support the classical view of a reticular or glial coating by GOLGI (1898, cited by LIPSKY, 1989) in the origin of perineuronal sulfated proteoglycans. Our previous double staining of human and mouse samples with silver nitrate and cationic iron colloid has shown that the Golgi's reticular coating stained with silver nitrate is identical to the perineuronal sulfated proteoglycans reactive to cationic iron colloid (MURAKAMI et al., 1996a, b).

Also noteworthy is the finding that in newborn animals, Golgi complexes of the neurons, whose cell surfaces are labeled with lectin Vicia villosa, soybean or Wisteria floribunda agglutinin, are labeled with these lectins. This finding suggests that the cell surface glycoproteins might be produced by the same nerve cells. No marked reaction of Golgi complexes of the neurons to the lectins after weaning indicates that the cell surface glycoproteins are also poorly supplied in adult animals.

In newborn and adult animals, no glial cells were labeled with lectin Vicia villosa, soybean and Wisteria floribunda. The present study thus offered no evidence supporting the view by BRÜCKNER et al. $(1993,1996)$, BLÜMCKE et al. (1995) and other authors (DEROUICHE et al., 1996) that the "perineuronal nets" labeled with lectin Vicia villosa, soybean or Wisteria floribunda agglutinin are produced by glial cells.

The present study, together with our previous result in the rat brain (TsubOUCHI, M. et al., 1996), indicates that the dark or active neurons in the cerebral cortex begin to appear 3-4 weeks after birth, i.e., closely before weaning. This implies that the cerebral cortex starts to function at this period.

Acknowledgments. We are grateful to Mr. Hiromichi Kusano, Mr. Masahiro NARASAKI and Mr. Hisao MizoGUCHI for tissue preparation and staining.

\section{REFERENCES}

Bertolloto, A., G. Rocca and D. Schiffer: Chondroitin 4-sulfate proteoglycans forms an extracellular networks in human and rat central nervous system. J. Neurol. Sci. 100: 113-123 (1990).

Bignami, A., R. Asher and G. Perides: Co-location of hyaluronic acid and chondroitin sulfate proteoglycans in rat cerebral cortex. Brain Res. 579: 173-177 (1992).

BlüMCKe, I., P. EgGLI and M. R. Celio: Relationship between astrocytic processes and "perineuronal nets" in rat neocortex. Glia 15: 131-140 (1995).

BraUer, K., G. BRÜCKNER, L. LEIBNITZ and L. WeINER: Structural and cytochemical features of perineuronal glial nets in the rat brain. Acta Histochem. 74: 53-60 (1984).

BrüCKNer, G., K. Brauer, W. Härtig, J. R. WolfF, M. J. Rickmann, A. Derouiche, B. Delpech, N. Gigard, W. H. Oertel and A. Reichenbach : Perineuronal nets provide a polyanionic, glia-associated form of microenvironment around certain neurons in many parts of the rat brain. Glia 8: 183-200 (1993).

Brückner, G., W. Härtig, J. Kacza, J. Seeger, K. WeLT and K. Brauer: Extracellular matrix organization in various regions of rat brain grey matter. J. Neurocytol. 25: 333-346 (1996).

Celio, M. R. and I. BLüMcke: Perineuronal nets. A specialized form of extracellular matrix in the adult nervous system. Brain Res. Rev. 19: 128-145 (1994).

Derouiche, A., W. HärtiG, K. Brauer and G. BRÜCKNER: Spatial relationship of lectin-labelled extracellular matrix and glutamine synthetase-immunoreactive astrocytes in rat cortical forebrain regions. J. Anat. 189: 363-372 (1996).

Drake, C. T., A. Mulligan., T. L. Wimpey, A. HendRICKSON and C. Chavkin: Characterization of Vicia villosa agglutinin-labeled GABAergic interneurons in the hippocampal formation and in acute dissociated hippocampus. Brain Res. 554: 176-185 (1991).

FujiTa, T.: Histological studies on the neuro-insular complex in the pancreas of some mammals. Z. Zellforsch. 50: 94-109 (1959).

GoLGI, C.: Intorno alla struttura delle cellule nervose. Arch. Ital. Biol. 30: 60-71 (1898).

Gomori, G.: Aldehyde-fuchsin: A new stain for elastic tissue. Amer. J. Clin. Pathol. 20: 665-666 (1950).

HÄrTIG, W., K. BRAUER and G. BRÜCKNER: Wisteria floribunda agglutinin-labeled nets surrond parvalbumincontaining neurons. Neuroreport 3: 869-872 (1992).

Härtig, W., K. Brauer, V. Vigl and G. BRÜCKNER: Chondroitin sulfate proteoglycan-immunoreactivity of lectin-labelled perineuronal nets around parvalbumincontaining neurons. Brain Res. 635: 307-311 (1994).

HendRY, S. H. C., E. G. Jones, S. Hockfield and R. D. G. MCKAY: Neuronal populations stained with the monoclonal antibody Cat-301 in the mammalian cerebral cortex and thalamus. J. Neurosci. 8: 518-542 (1988). 
Kosaka, T. and C. W. Heizmann : Selective staining of a population of parvalbumin-containing GABAergic neurons in the rat cerebral cortex by lectins with specific affinity for terminal $N$-acetylgalactosamine. Brain Res. 483: 158-163 (1989).

LIPSKY, N. G.: On the structure of nerve cells, by C. Golgi. Historical note. J. Microsc. 155: 3-7 (1989).

LÜth, H.-J., L. Fischer and M. R. Celio: Soybean lectin binding neurons in the visual cortex of the rat contain pravalbumin and are covered by glial nets. J. Neurocytol. 21: 211-221 (1992).

MuRAKaMi, T.: Strongly negatively charged extracellular matrix in the mouse central nervous system (Japanese text with English abstract). Okayama Igakkai Zasshi 106: 1063-1071 (1994).

Murakami, T. and A. Ohtsuka: Sleep restores the ratio of dark neurons to light neurons in the central nervous system (Japanese text with English abstract). Okayama Igakkai Zasshi 107: 235-242 (1996).

Murakami, T., T. Taguchi, A. Ohtsuka, K. Sano, T. Kaneshige, R. L. OWen and A. L. Jones: A modified method of fine-granular cationic iron colloid priparation: Its use in the light and electron microscopic detection of anionic sites in the rat kidney glomerulus and certain other tissues. Arch. Histol. Cytol. 49: 12-23 (1986).

Murakami, T., T. Taguchi, A. Ohtsuka and A. KikUta: Neurons with strongly negative- charged surface- coats in adult rat brain as detected by staining with cationic iron colloid. Arch. Histol. Cytol. 56: 13-21 (1993a).

Murakami, T., T. Taguchi and A. Ohtsuka: The occurrence in the human brain of neurons with strongly negative-charged proteoglycans. Arch. Histol. Cytol. 56: 23-26 (1993b).

Murakami, T., Y. Tsubouchi, M. Tsubouchi, A. Ohtsuka, T. TAguchi and A. Kinuta: The occurrence of rat spinal cord neurons with strongly negative-charged surface coats. Arch. Histol. Cytol. 56: 501-504 (1993c).

Murakami, T., M. Tsubouchi, Y. Tsubouchi, T. Taguchi and A. OHTsuka: The occurrence of neurons with strongly negatively charged surface coats in mammalian, avian, reptilian, amphibian and piscine brains. Acta Med. Okayama 48: 195-197 (1994a).

Murakami, T., A. Ohtsuka and T. Taguchi: Neurons with intensely negatively charged extracellular matrix in the human visual cortex. Arch. Histol. Cytol. 57: 509522 (1994b)

Murakami, T., S. Hitomi, A. OHtsuka and T. Taguchi: Neurons with perineuronal sulfated proteoglycans in the human visual cortex, with special reference to their reactions to lectins. Arch. Histol. Cytol. 58: 357-364 (1995a).

Murakami, T., A. Ohtsuka, T. Taguchi and D. X. Piao: Perineuronal sulfated proteoglycans and dark neurons in the brain and spinal cord: A histochemical and electron microscopic study of newborn and adult mice. Arch. Histol. Cytol. 58: 557-565 (1995b).

Murakami, T., A. Ohtsuka and K. Ono: Neurons with perineuronal sulfated proteoglycans in the mouse brain and spinal cord: Their distribution and reactions to lectin Vicea villosa agglutinin and Golgi's silver nitrate. Arch. Histol. Cytol. 59: 219-231 (1996a).

Murakami, T., A. Ohtsuka and D. X. Pia0: Perineuronal sulfated proteoglycans in the human brain are identical to Golgi's reticular coating. Arch. Histol. Cytol. 59: 233-237 (1996b).

Murakami, T., T. Murakami, N. Mahmut and A. OHTSUKA: Dark and light neurons in the human brain, with special reference to their reaction to Golgi's silver nitrate, luxol fast blue MBS and azocarmine G. Arch. Histol. Cytol. 60: 265-274 (1997a).

Murakami, T., W. D. Su, L. J. Hong, D. X. Piao, A. OHTSUKA and K. SEno: Demonstration of tissue-reacted cationic iron colloid with protein silver and gold chloride (Japanese text with English abstract). Okayama Igakkai Zasshi 109: in press (1997b).

NaEgele, J. R. and L. C. KATz: Cell surface molecules containing $\mathrm{N}$-acetylgalactosamine are associated with basket cells and neurogliaform cells in cat visual cortex. J. Neurosci. 10: 540-557 (1990).

Nakagawa, F., A. Schulte and S. S. SpICer: Selective cytochemical demonstration of glycoconjugate-containing terminal $N$-acetylgalactosamine on some brain neurons. J. Comp. Neurol. 243: 280-290 (1986).

Ohtsuka, A. and T. Murakami: Dark neurons in the mouse brain: An investigation into possible significance of their variable appearance within a day and their relation to negatively charged cell coats. Arch. Histol. Cytol. 59: 79-85 (1996).

Ruoslahti, E.: Brain extracellular matrix. Glycobiology 6: 489-492 (1996).

Seeger, G., K. BraUer, W. Härtig and G. BrüCKNer: Mapping of perineuronal nets in the rat brain stained by colloidal iron hydroxide histochemistry and lectin cytochemistry. Neuroscience 58: 371-388 (1994).

Tsubouchi, M., Y. Tsubouchi, S. Hitomi, A. Ohtsuka and T. Murakami: Perineuronal sulfated proteoglycans, cell surface glycoproteins and dark neurons in the cingulate cortex of the newborn and adult rats. Acta Med. Okayama 50: 313-317 (1996).

Tsubouchi, Y., M. Tsubouchi, S. Нiтomi, А. Оhтsuka and T. MURAKAMI: Perineuronal sulfated proteoglycans in the adult rat brain: Histochemical and electron microscopic studies. Acta Med. Okayama 50: 237-241 (1996). 